\title{
Compact Slot and Dielectric Resonator Antenna With Dual-Resonance, Broadband Characteristics
}

\author{
Amelia Buerkle, Student Member, IEEE, Kamal Sarabandi, Fellow, IEEE, and Hossein Mosallaei, Senior Member, IEEE
}

\begin{abstract}
The goal of this study is to improve the bandwidth of a miniaturized antenna. The proposed technique combines a slot antenna and a dielectric resonator antenna (DRA) to effectively double the available bandwidth without compromising miniaturization or efficiency. With proper design it is observed that the resonance of the slot and that of the dielectric structure itself may be merged to achieve extremely wide bandwidth over which the antenna polarization and radiation pattern are preserved. In addition, using the DRA, a volumetric source, improves the radiation power factor of the radiating slot. A miniaturized antenna figure of merit (MAFM) is defined to simultaneously quantify aspects of miniaturized antenna performance including the degree of miniaturization, efficiency, and bandwidth. Figures for various common types of antennas are given and compared with that of the proposed structures. In order to determine the effects of varying design parameters on bandwidth and matching, sensitivity analysis is carried out using the finite-difference time-domain method. Numerous designs for miniaturized slot-fed dielectric resonator antennas are simulated and bandwidths exceeding $25 \%$ are achieved. Two 2.4 GHz antennas are built, characterized, and the results compared with theory.
\end{abstract}

Index Terms-Dielectric resonator antenna (DRA), dual-resonance, slot antenna.

\section{INTRODUCTION}

A NTENNA miniaturization is becoming increasingly important especially in wireless and low frequency applications. The demand for wireless technology has increased dramatically in the past decade. Antennas which are small, efficient, and integrable into mobile devices must be developed. Alternatively, as the frequency of operation is lowered, miniaturization techniques must be employed to keep antenna size practical. There are two primary techniques to achieve antenna miniaturization. The first is using novel topology to reduce the overall area consumed by the radiating structure. The second is using materials with permittivity, permeability, or both, greater than one; since wavelengths inside such materials are reduced, the characteristic size of the antenna decreases. This study uses the latter technique to achieve antenna miniaturization by employing a dielectric resonator as a superstrate for the slot antenna as shown in Fig. 1. This increases the effective permittivity seen by the slot and lowers its resonant frequency.

Truncating the dielectric superstrate yields a resonant structure called a dielectric resonator antenna (DRA). Dielectric resonator antennas are experiencing increased use in antenna ap-

Manuscript received November 25, 2003; revised April 7, 2004.

The authors are with the Radiation Laboratory, Electrical Engineering and Computer Science Department, University of Michigan, Ann Arbor, MI 48109-

2122 USA (e-mail: saraband@eecs.umich.edu).

Digital Object Identifier 10.1109/TAP.2004.842681
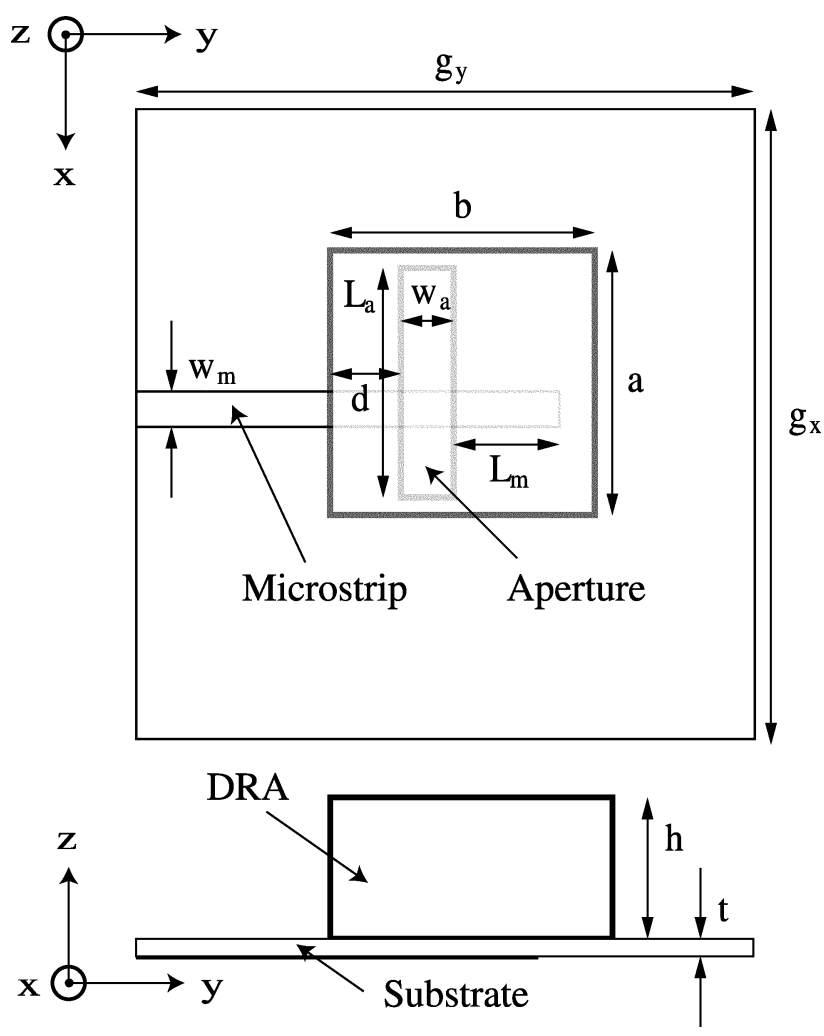

Fig. 1. Diagram of antenna design. The dielectric resonator is coupled to a microstrip-fed slot in the ground plane.

plications due to favorable properties including low cost, inherently wide bandwidth, small size, and high efficiency due to the absence of conductors and surface wave losses. They are simple to integrate into existing fabrication technology and have a flexible design process with numerous design parameters, such as shape, size, aspect ratios, and permittivity. The current technique improves the bandwidth of these antennas without compromising their efficiency and other positive characteristics.

It is known that miniaturization directly affects an antenna's ability to radiate. In [1] Wheeler constructs the radiation power factor (RPF) to quantify the radiation properties of electrically small antennas. The value, which is inversely related to $Q$, indicates the amount of real power radiated away from the antenna relative to the amount of reactive power which is stored in the antenna's near field. Wheeler derives a formulation of the RPF in terms of the volume of the antenna:

$$
P F \propto \frac{V_{a}}{V_{s}}
$$

where $V_{a}$ represents the effective antenna volume, which is related to its physical volume, and $V_{s}$ the volume of the radian 
sphere in terms of the free-space operational wavelength, $V_{s}=$ $(4 \pi / 3)(\lambda / 2 \pi)^{3}$. Often miniaturized antennas, especially linear or surface radiators, which have small effective volumes exhibit poor radiation capability and tend to store energy in the near field surrounding them. For a volumetric source, such as the dielectric resonator, the RPF is improved because $V_{a}$ is much larger. The dielectric resonator can be modeled by an equivalent volumetric current source of the form

$$
\overrightarrow{J_{v}}=j \omega \epsilon_{o}\left(\epsilon_{r}-1\right) \vec{E}
$$

where $\epsilon_{r}$ is the relative permittivity of the resonator and $\vec{E}$ is the electric field inside the resonator. The DRA is a good radiator because it fills the radian sphere surrounding it, making $V_{a} \approx V_{s}$ depending on frequency. In the same way, the DRA improves the radiation power factor of the underlying slot antenna at its radiation frequency by more effectively filling its radian sphere, i.e., it fills the near field of the slot antenna, increasing its effective volume and the amount of real power radiated away from the antenna into the far field.

As the primary goal of this study is to improve bandwidth while maintaining small size, the relationship between the maximum possible bandwidth of an antenna relative to its size must be investigated. In 1948, Chu derived fundamental limitations on achievable bandwidth for a small antenna, or one which fits inside the radian sphere. His results show that as size is reduced, the maximum possible bandwidth decreases. If a multi-resonant structure is used these limitations, while qualitatively relevant, do not directly apply. This is justifiable by noting that in filter design many high-Q resonators can be used to achieve the desired wide bandwidth of operation. By applying this technique of merging the slot and DRA resonances bandwidths approaching and surpassing Chu's limit may be obtained. It is noted that the DRAs used in this study have moderate permittivity $\left(\epsilon_{r}=12\right)$ and do not adhere to the strict definition of a small antenna over the entire bandwidth. Using a higher permittivity DRA, however, in similar designs would allow the bandwidth to approach Chu's limit.

A miniaturized antenna figure of merit (MAFM) is defined to facilitate comparison between miniaturized antennas' performance. Comparison between antennas becomes difficult when size is reduced and other characteristics must be considered. This is especially true when multiple resonances are used to enhance bandwidth because $\mathrm{Q}$ becomes less well-defined. The MAFM is defined as follows:

$\operatorname{MAFM}_{\mathrm{dB}}=10 \log _{10}\left(\eta \cdot \frac{\Delta f}{f_{c}} \cdot \frac{\lambda}{D}\right)=\eta_{\mathrm{dB}}+\left(\frac{\Delta f}{f_{c}}\right)_{\mathrm{dB}}+\left(\frac{\lambda}{D}\right)_{\mathrm{dB}}$

where $\eta$ is the antenna efficiency relating its gain and directivity, $\Delta f$ is the $-10 \mathrm{~dB}$ bandwidth, $f_{c}$ is the center frequency, $D$ is the largest linear dimension of the antenna, and $\lambda$ is the freespace wavelength. By simultaneously accounting for the efficiency, bandwidth, and miniaturization, the MAFM is a more comprehensive means of comparison between antennas. A metallic $\lambda / 2$ dipole with $5 \%$ bandwidth and $99 \%$ efficiency, for example, has a MAFM of $-0.04 \mathrm{~dB}-13 \mathrm{~dB}+3 \mathrm{~dB}=-10.04 \mathrm{~dB}$. A 10 $\mathrm{GHz}$ patch antenna fabricated on duroid $\left(\epsilon_{r}=2.2\right)$ with $1 \%$ bandwidth and $86 \%$ efficiency has a MAFM of $-15.9 \mathrm{~dB}$. In Section IV, the MAFM for the proposed antennas is computed from measured results and is found to be near $0 \mathrm{~dB}$.

Numerous studies on dielectric resonator antennas have been carried out. It is interesting to note that previous studies on DRAs neglect constructive use of the feed resonance which is inherently present in the system. McAllister, et al., mention the presence of the probe resonance in [2] but do not address its usefulness in achieving wide bandwidths. Designing the probe feed to optimize the bandwidth of the DRA resonance has been noted in [3], [4]; however, the probe's own resonance is not used. In this study, a probe feed was initially used to feed the DRA. However, the probe length was limited by the dielectric size and matching was sometimes difficult to achieve. In addition, the probe possesses large self-reactance at high frequencies and is a source of ohmic loss [5]. A slot feed was adopted to simplify fabrication and improve matching to a $50 \Omega$ microstrip line. Low cross-polarization levels are also maintained by using a slot feed because both the slot and the DRA radiate as horizontal magnetic dipoles. Many other studies use a nonresonant slot to feed the dielectric resonator [6]-[9]. As discussed, since multiple resonance systems are not subject to typical bandwidth limitations that afflict electrically small antennas, Chu's lower bound on $\mathrm{Q}$ is not a limiting factor on the bandwidth of the proposed structures.

The technique of merging the resonance of the feed mechanism with that of the radiating structure has been employed with other types of antennas, specifically aperture-fed microstrip patch antennas [10]. The benefits of DRAs over existing microstrip antennas which include wider bandwidth, higher efficiency, and more feeding options, encourage the extension of this principle to DRAs. Wideband DRAs have also been under development for some time; a review of many current designs may be found in [11]. Often resonator geometry is altered to maximize bandwidth. For example, [3], [4], [7], [12] study the impedance and radiation characteristics of triangular, conical, tetrahedral, and split cylindrical resonator geometries; $50 \%$ impedance-bandwidth using split cylindrical resonators has been reported. Notched rectangular DR structures have also been used to obtain impedance-bandwidths up to 28\% [13] and multi-segment DRAs (MSDRA), in which layers of high permittivity dielectric are placed beneath the DRA to improve matching to the microstrip feed, offer bandwidths up to $20 \%$ [14]. These are more complex designs, however, and are more difficult to fabricate than the current method. It is noted that the proposed technique also has nonnegligible back radiation making these designs more appropriate in applications which require a high front-to-back ratio.

Refinements in the design are carried out using the finite-difference time-domain (FDTD) method. The effects of varying design parameters are investigated in a parametric study, the results of which are given in Section III. Knowledge from this analysis is used to merge the two resonances in further design simulations. Two designs with bandwidths exceeding $25 \%$ are built; previous designs based on a single resonance and of comparable structural complexity exhibit much smaller bandwidths.

The organization of the paper is as follows. In Section II the antenna configuration is presented and the design variables are 
reviewed. In Section III a detailed parametric study is summarized and observed trends are given to aid in the design procedure. Finally, in Section IV measurements of fabricated designs are given and compared with simulation results.

\section{CONCEPT AND DESIGN}

The DRA and slot are both resonant structures. Together they yield a double resonant structure with low cross-polarization levels and identical radiation patterns. With proper design, the two resonances can be merged to acheive wide bandwidth. The design process is initiated with a choice of the desired frequency of operation. The corresponding size of resonator can be approximated using various methods some of which are evaluated in [15], [16]. In this study the conventional dielectric waveguide model (CDWM) is used. The resonant frequency of the slot can be approximated using the length of the slot and the permittivity of the dielectric resonator. In general, the relative frequencies of the resonances are particular to the design. In this study the lower resonance is associated with the feed mechanism while the upper resonance results from the resonator itself.

The DR is coupled to a microstrip-fed slot in the ground plane, as shown in Fig. 1. To a first order approximation the DRA and slot resonance may be designed independently of each other. However, in reality the resonances are coupled and even minor parameter variations affect loading and matching at both resonances. There are many parameters which can be varied to achieve matching and wide bandwidth; these include, among others, slot dimension, relative position of the slot and the DRA, and microstrip parameters. In this section, the design variations employed in the study are evaluated from a theoretical point of view. The effect of slot position and size on resonance location and matching will first be discussed; then the DRA parameters will be evaluated. A more detailed review of the observed effects of varying single parameters in simulation is given in the next section.

The size and position of the slot are varied in order to improve matching and control resonance frequency. The slot resonance occurs at approximately $\lambda_{g} / 2$, where $\lambda_{g}=\lambda_{o} / \sqrt{\mu_{\text {eff }} \epsilon_{\text {eff }}}$ is the wavelength inside the dielectric material. The slot sees an effective permittivity

$$
\epsilon_{\text {eff }}=\frac{H_{\text {total }}}{\frac{h}{\epsilon_{\text {dra }}}+\frac{t}{\epsilon_{s s}}}
$$

[14], where $H_{\text {total }}$ is the total height of the antenna structure, $h$ is the height of the DRA with permittivity $\epsilon_{\mathrm{dra}}$, and $t$ is the thickness of the substrate of permittivity $\epsilon_{s s}$. Other design parameters affect the slot resonance frequency as well but to a lesser degree. The width of the slot is kept small relative to the wavelengths of operation; therefore, the electric field is primarily in the $\hat{y}$ direction. The slot can be modeled by a magnetic current source, $\vec{M}=-\hat{z} \times E_{a} \hat{y}$, where $E_{a} \hat{y}$ is the electric field in the slot induced by currents on the ground plane. In this case a magnetic current source lies along the $\hat{x}$ direction along the bottom of the DRA.

It has been shown that the slot and antenna appear as a series load along a transmission line which is terminated in an open
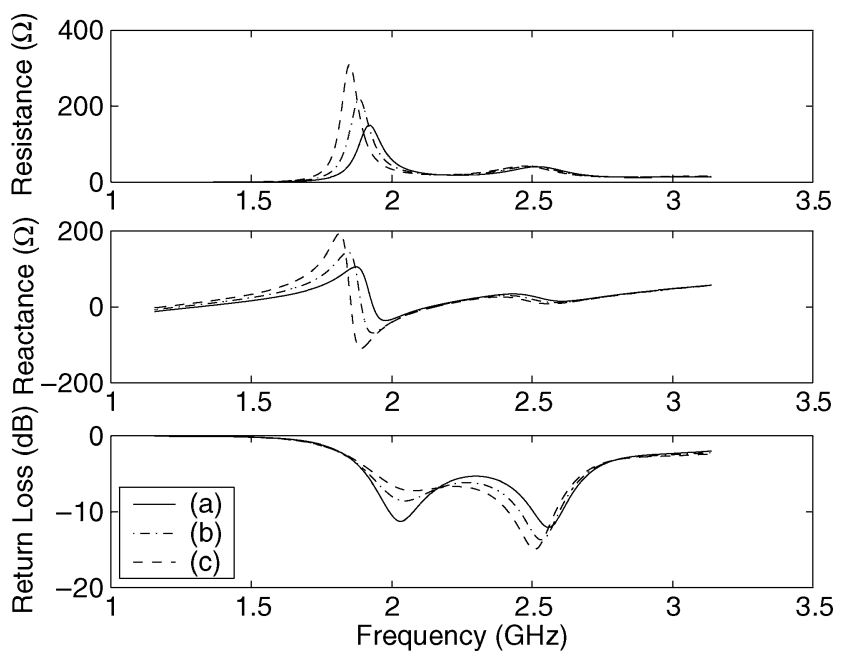

Fig. 2. Length of the microstrip is varied. $L_{s}=2.33 \mathrm{~cm}, w_{s}=0.5 \mathrm{~cm}$, and $d=0.33 \mathrm{~cm}$. (a) $L_{m}=0.67 \mathrm{~cm}$. (b) $L_{m}=0.83 \mathrm{~cm}$. (c) $L_{m}=1 \mathrm{~cm}$.

circuit at the end of the microstrip [17]. Matching is achieved when the input reactance is completely compensated for and the input impedance has only a resistive component. The reactive portion of the input impedance can be compensated for using the appropriate length of microstrip line which acts as an open stub tuner. It is observed in Fig. 2 the microstrip length plays a minor role in resonance location as well. However, as microstrip length affects matching this is a difficult parameter with which to control resonances beyond minor adjustments. Moving the slot in the $\hat{y}$ direction, which in effect changes the length of the microstrip but also affects coupling to the DRA, is observed to move the resonances more effectively as seen in Fig. 5.

The real part of the input impedance is affected by moving the slot in the $\hat{x}$ direction, perpendicular to the microstrip. The electric field in the slot, $E_{a} \hat{y}$, and the resulting equivalent magnetic current source, must go to zero at the ends of the slot due to the boundary condition on tangential components of the electric field. The current, $\vec{J}$, on the ground plane does the reverse, reaching its maximum around the edges of the slot and going to zero in the middle. The resistance varies as the ratio of the magnetic current to the physical electric current over the length of the slot; therefore, by placing the slot at the correct $\hat{x}$ location matching to any resistance can theoretically be realized. Movement along the $\hat{x}$ direction is not observed to affect resonance location. In this study the DRA is moved with the slot, and remains covering the slot where possible.

The approximate expressions for each field component inside the resonator may be obtained from the electric vector potential and the boundary conditions for the CDWM. The formulas are here reviewed in brief; additional information may be found in [6]. With reference to Fig. 1, and taking the origin at the center of the DRA, perfect magnetic walls are assumed to lie in the $x-z$ plane at $y=-b / 2$ and at $y=b / 2$, and in the $y-z$ plane at $x=-a / 2$ and $x=a / 2$. Continuity of the tangential fields is enforced at $z=h$ and, by image theory, at $z=-h$. The electric vector potential, $\vec{F}$, for the $T E_{111}^{z}$ mode is then given by

$$
\vec{F}=F_{z} \hat{z}=A \cos \left(k_{x} x\right) \cos \left(k_{y} y\right) \sin \left(k_{z} z\right)
$$


where $A$ is a constant and the wavenumbers are as follows [18]:

$$
k_{x}=\frac{n \pi}{a} \quad k_{y}=\frac{m \pi}{b} \quad k_{z} \tan \left(k_{z} h\right)=\frac{\mu_{\mathrm{dra}}}{\mu_{o}} \sqrt{k_{x}^{2}+k_{y}^{2}-k_{o}^{2}}
$$

where $k_{o}$ is the free space wavenumber, $\mu_{\mathrm{dra}}$ is the permeability of the DRA, and $k_{x}, k_{y}, k_{z}$ are the wavenumbers in the $x, y$, and $z$ directions, respectively. By replacing $\mu$ with $\epsilon$ in these equations, analogous expressions for TM modes may be found. The wavenumbers satisfy the following characteristic equation:

$$
k_{x}^{2}+k_{y}^{2}+k_{z}^{2}=\mu_{r, \mathrm{dra}} \epsilon_{r, \mathrm{dra}} k_{o}^{2}
$$

where $\mu_{r, \text { dra }}$ and $\epsilon_{r, \text { dra }}$ are the relative permeability and permittivity of the medium and the remaining variables are the same as above. These equations may be used to relate size with operational frequency. For example, the fabricated DRA discussed in Section IV has dimensions $a=b=2.67 \mathrm{~cm}$ and $h=1.67 \mathrm{~cm}$ resulting in a resonant frequency of $2.49 \mathrm{GHz}$, which is within $5 \%$ of the measured value.

Assuming matching to the resonator is adequate the resulting magnetic dipole current source lies along the $\hat{x}$ direction and excites the $T E_{111}^{x}$ mode of the DRA. In reality, this is a hybrid mode so $E_{x}$ is small but nonzero.

Consideration should be given to the choice of material permittivity to use in the DRA; along with operational frequency this parameter determines the antenna size. High permittivity materials, while allowing smaller antenna size, make it more difficult to merge resonances as they result in narrower bandwidths. As shown in [6], the quality factor, $Q \propto \epsilon_{r}^{3 / 2}$, where $\epsilon_{r}$ is the relative permittivity of the resonator; therefore, bandwidth which is inversely related to $Q$ decreases as permittivity increases. The DRA permittivity also determines the effective permittivity seen by the slot, effecting its resonant frequency and the input impedance as well [9].

As expected, ground plane size significantly affects the results. In Section IV, for example, the second fabricated antenna on the $8 \mathrm{~cm}$ ground plane was adopted from the first design on a smaller ground plane. The design parameters of the second design had to be changed significantly in order to achieve matching and merge the resonances.

\section{PARAMETRIC STUDY}

This section gives the observed simulation results when the parameters discussed in Section II are systematically varied. Simulations are carried out using the finite-difference time-domain approach [19]. In reference to Fig. 1, $a$ and $b$ are the $x$ and $y$ dimensions of the dielectric resonator, respectively, and $g_{x}$ and $g_{y}$ are those of the ground plane. The height of the DRA is denoted by $h$ and the thickness of the substrate by $t$. The microstrip line has width $w_{m} ; L_{m}$ is the microstrip length beyond the slot. $L_{s}$ is the slot length and $w_{s}$ is the width. The distance from the edge of the DRA to the edge of the slot on the feed side is $d$. Throughout this analysis the DRA is centered over the slot along the $x$ direction.

All of the simulated designs in this section, except where otherwise stated, have dielectric resonators that are $2.67 \mathrm{~cm}$ square in the $x-y$ plane and $1.67-\mathrm{cm}$ tall; a permittivity of 12 is used

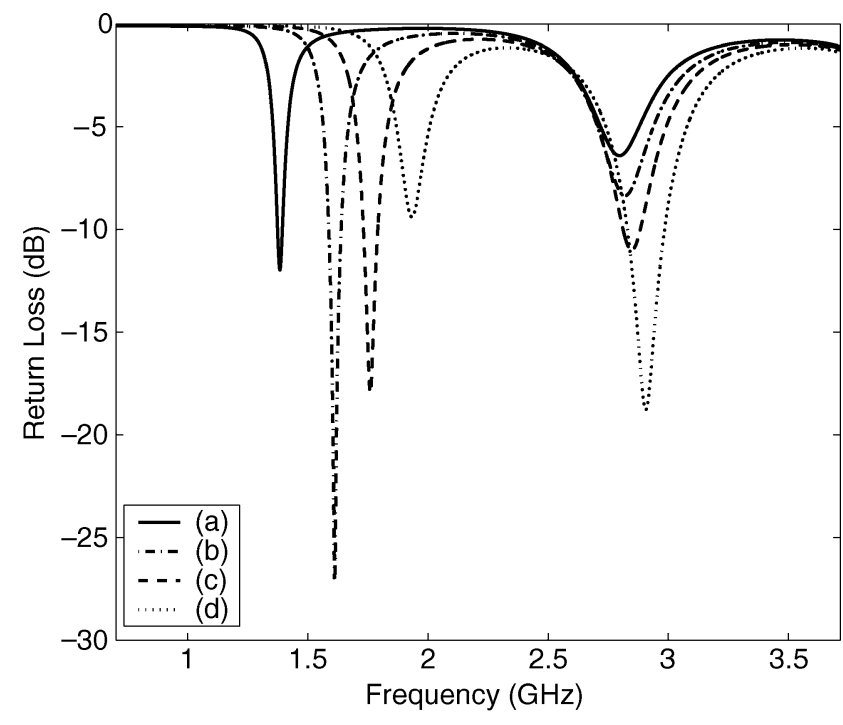

Fig. 3. Length of the slot is varied. $w_{s}=0.5 \mathrm{~cm}, d=1 \mathrm{~cm}, L_{m}=$ $0.167 \mathrm{~cm} \mathrm{(a)} L_{s}=3.33 \mathrm{~cm}, 0.33 \mathrm{~cm}$ longer on both ends of the DRA. (b) $L_{s}=2.67 \mathrm{~cm}$, the same size as the DRA. (c) $L_{s}=2.33 \mathrm{~cm}, 0.167 \mathrm{~cm}$ shorter than the DRA at both ends. (d) $L_{s}=2 \mathrm{~cm}, 0.33 \mathrm{~cm}$ shorter than the DRA at both ends.

with a loss tangent of $5.6 e-4$. The ground plane is $5.66-\mathrm{cm}$ square with a substrate thickness of $0.167 \mathrm{~cm}, \epsilon_{r}=2.2$, and $\tan \delta_{e}=5.6 e-4$. The microstrip feeding the slot is $0.5-\mathrm{cm}$ wide; its length varies with the design.

\section{A. Slot Dimensions}

In Fig. 3, the variation of return loss is shown as the slot length, $L_{s}$ (see Fig. 1), is varied from 3.33 to $2 \mathrm{~cm}$ while the width, $w_{s}$, is maintained at $0.5 \mathrm{~cm}$. All other parameters such as resonator size and position are kept constant. In the first case the slot extends beyond the dielectric for $0.33 \mathrm{~cm}$ on each end; in the last three designs it remains covered by the DRA. The upper resonance mainly stays fixed at the same frequency while the lower resonance shifts up with decreasing slot length. Similar results are obtained when the slot width, $w_{s}$, is varied from 0.33 to $0.67 \mathrm{~cm}$ while the length is maintained at $2.33 \mathrm{~cm}$. In agreement with [20], slot width has a smaller effect on matching than slot length; however, it too affects the lower resonance. These results provide evidence that the lower frequency resonance is due to radiation from the coupling slot.

\section{B. Resonator Dimensions}

In Fig. 4, the dimensions of the DRA are varied; all designs have a $6 \mathrm{~cm}$ square ground plane, dielectric resonator height $1.67 \mathrm{~cm}$, and slot dimensions 2 by $0.5 \mathrm{~cm}$. The result from a 2.67-cm square DRA is shown in (a). In the structure in curve (b), $a=3.34 \mathrm{~cm}$ and $b$ remains $2.67 \mathrm{~cm}$ in $y$. In that of curve (c), $b=3.34 \mathrm{~cm}$ and $a=2.67 \mathrm{~cm}$. Finally, (d) corresponds to a $3.34 \mathrm{~cm}$ square resonator. Fig. 4 also demonstrates that the $T E_{111}^{x}$ mode is excited by the slot which is expected given the orientation of the slot in the ground plane. Similar results are obtained when the DRA height, $h$, is varied while $a$ and $b$ remain $2.67 \mathrm{~cm}$ each. From this evidence it is concluded that the upper resonance is predominantly effected by the dielectric resonator dimensions. 


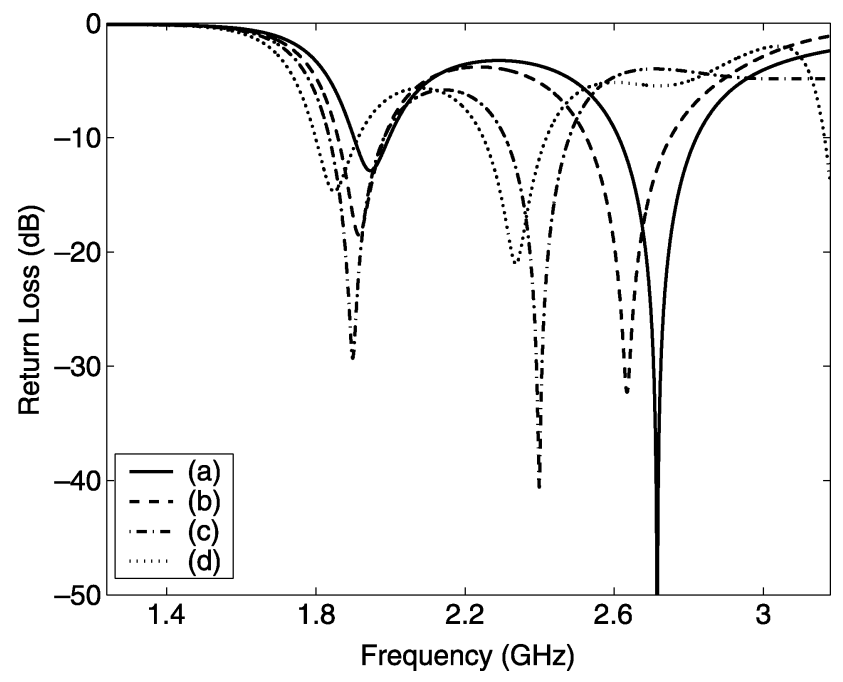

Fig. 4. $x$ and $y$ dimensions of the DRA are varied. The height $h$, remains at $1.67 \mathrm{~cm}, L_{s}=2 \mathrm{~cm}$, and $w_{s}=0.5 \mathrm{~cm}$. (a) $a=b=2.67 \mathrm{~cm}$. (b) $a=$ $3.34 \mathrm{~cm}, b=2.67 \mathrm{~cm}$. (c) $a=2.67 \mathrm{~cm}, b=3.34 \mathrm{~cm}$. (d) $a=b=3.34 \mathrm{~cm}$. The ground plane is $6-\mathrm{cm}$ square.
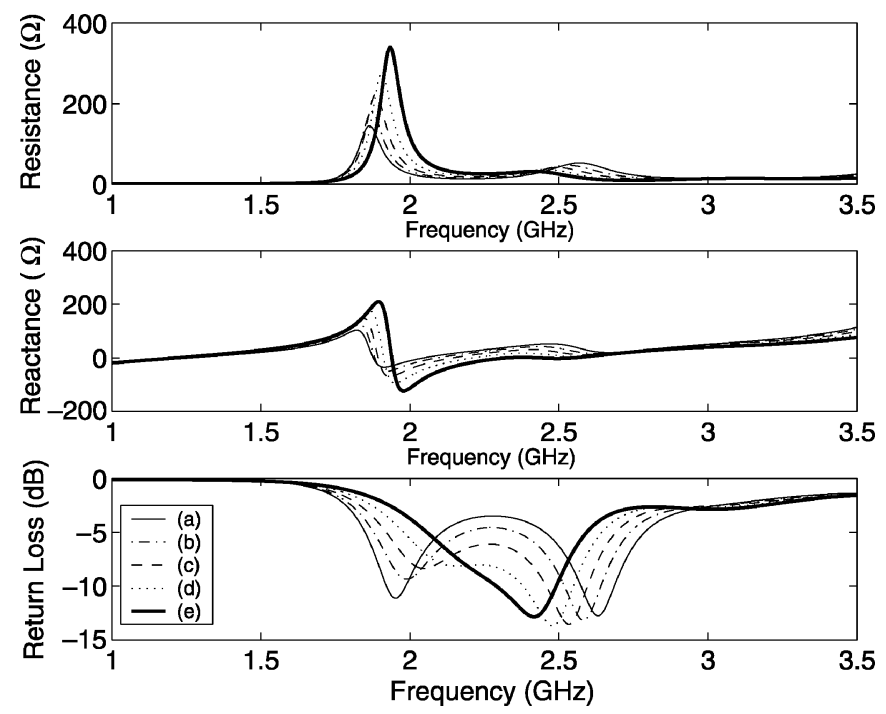

Fig. 5. Slot, $L_{s}=2 \mathrm{~cm}, w_{s}=0.5 \mathrm{~cm}$, is moved back along the microstrip in the $-y$ direction. (a) $d=0.5 \mathrm{~cm}, L_{m}=0.67 \mathrm{~cm}$. (b) $d=0.42, L_{m}=0.75$. (c) $d=0.33 \mathrm{~cm}, L_{m}=0.83 \mathrm{~cm}$. (d) $d=0.25 \mathrm{~cm}, L_{m}=0.92 \mathrm{~cm}$. (e) $d=0.167 \mathrm{~cm}, L_{m}=1 \mathrm{~cm}$.

\section{Microstrip Length}

In addition to affecting the reactive portion of the input impedance the length of the microstrip also influences the relative position of the resonances. Increasing its length is observed to merge the two resonances while decreasing it tends to separate them. Fig. 2 illustrates the effect of microstrip length on input impedance and resonance location. As the microstrip length is increased both the input resistance and reactance associated with the first resonance increase resulting in poorer impedance matching.

Fig. 5 shows the result if the position of the slot is moved back along the microstrip, corresponding to changing $d$ in Fig. 1; this is similar to increasing the length of the microstrip however the interaction between the DRA and the slot is affected as well. In simulation moving the slot back along the microstrip effected

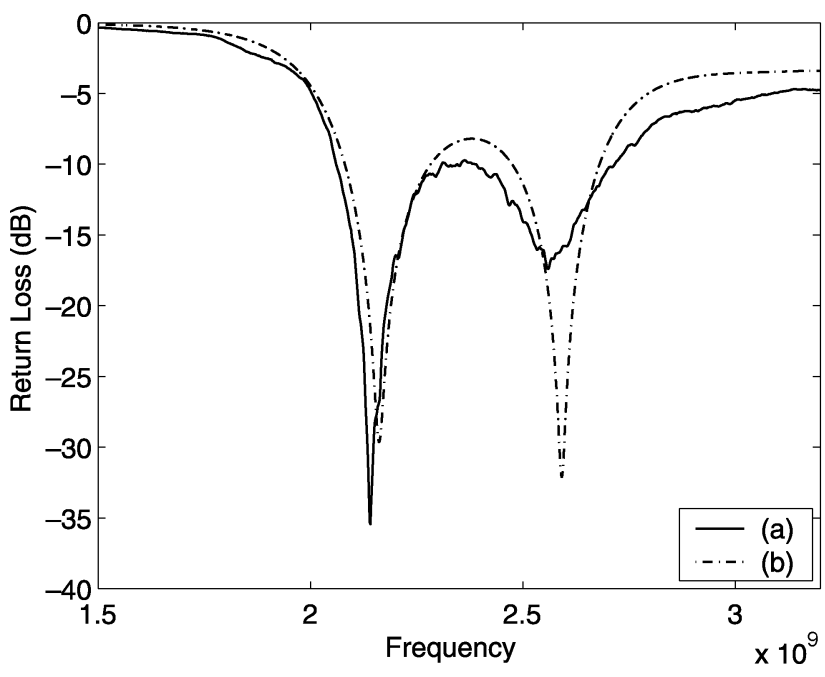

Fig. 6. Return loss for the $5.5 \mathrm{~cm}$ ground plane design (a) measured (b) simulated.

the relative resonance positions more than altering the length of the microstrip alone. In all cases shown the slot remains under the DRA, which is left in its original position. Note that the resonances could be over-merged, as in the last case in Fig. 5, however, separating them leads to larger bandwidths.

\section{MEASURED RESUlTS}

Two antennas similar to those simulated above are fabricated and measured. In both cases, the DRA, made from TransTech's SMAT12, is $2.67 \mathrm{~cm}$ square by $1.67 \mathrm{~cm}$ tall and have $\epsilon_{r}=12$ with $\tan \delta_{e}<1.5 e-4$. The substrate is 1.575 -mm thick Rogers RT5880 $\left(\epsilon_{r}=2.2, \tan \delta_{e}=4-9 e-4\right)$. The DRA and slot are offset in the $-x$ direction perpendicular to the microstrip; the slot remains centered in $x$ under the DRA. The first antenna is on a $5.5-\mathrm{cm}$ square ground plane while the second is on an $8-\mathrm{cm}$ square. The larger ground plane design was made to improve the radiation patterns and the front-to-back ratio.

For the antenna with the $5.5-\mathrm{cm}$ square ground plane, the microstrip is $0.5-\mathrm{cm}$ wide, $L_{m}=0.33 \mathrm{~cm}$, and $d=0.25 \mathrm{~cm}$. The slot is $2-\mathrm{cm}$ long by $0.5-\mathrm{cm}$ wide. The simulated and measured return losses are shown in Fig. 6. The measured $10 \mathrm{~dB}$ bandwidth is $24.7 \%$. The lack of agreement between the return losses of the simulated and measured designs may be due to an air gap between the ground plane and the resonator which is absent in simulations. The effect of such an air gap is studied extensively in [21]-[23]. Also, the simulation parameters and lengths are limited by the resolution of the FDTD grid. Therefore, they are not always equal to the physical antenna dimensions.

The radiation patterns in the $\mathrm{E}$ and $\mathrm{H}$ planes at the DRA resonance are shown in Fig. 7. The simulated patterns are shown as well; the bold lines correspond to the measurements. Since both the slot and the lowest order modes of the DRA radiate as horizontal magnetic dipoles, low cross-pol levels are maintained. Lower cross-pol levels are possible using a slot which is centered under the DRA in the $\hat{y}$ direction; this would mitigate the excitation of other resonator modes which contribute to cross-pol levels. The E-plane asymmetry is primarily an effect of the presence of the coaxial feed on the side of the ground 


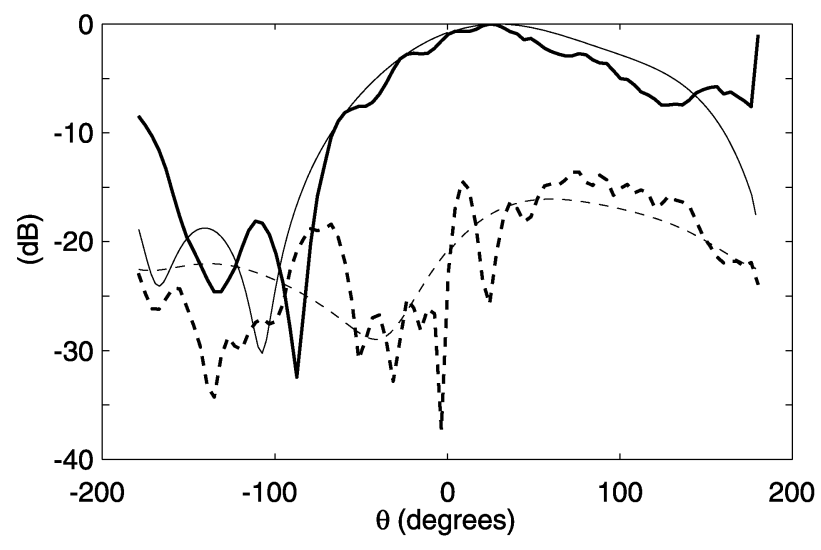

(a)

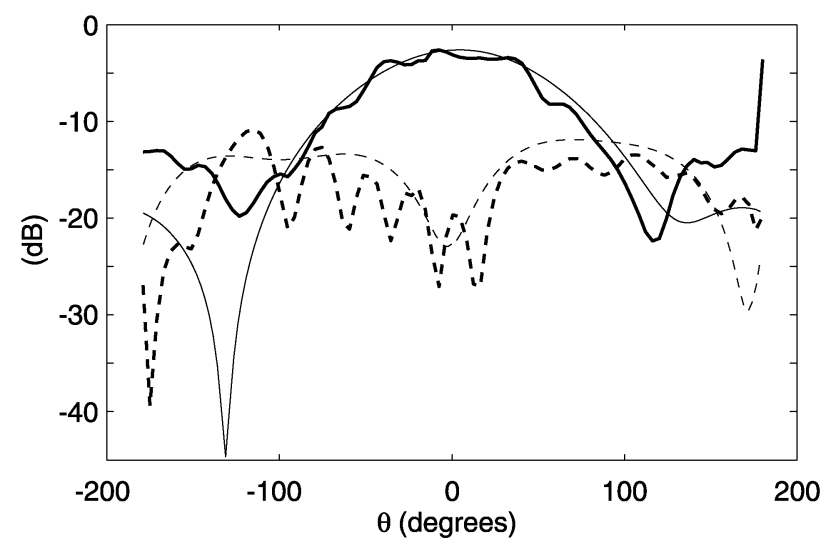

(b)

Fig. 7. E-plane and H-plane antenna patterns for the $5.5 \mathrm{~cm}$ ground plane design at the DRA resonance of $f=2.5 \mathrm{GHz}$. Measurements are shown in bold and simulations in regular line weight. (a) E-plane and (b) H-plane.

plane. The poor H-plane pattern symmetry and large back radiation are mostly a result of the small size of the ground plane. The larger, $8 \mathrm{~cm}$ design patterns are much improved. In addition, the possible presence of other resonator modes due to the offset slot contributes to pattern asymmetries which are still present in the $8 \mathrm{~cm}$ case.

The gain at the lower and upper resonance frequencies is approximately 4.6 and $2.8 \mathrm{dBi}$, respectively. Using $D=2.7 \mathrm{~cm}$, the resonator size, the MAFM becomes $1.05 \mathrm{~dB}$ at the lower resonance and $-0.19 \mathrm{~dB}$ at the upper resonance. These values demonstrate that the design maintains reasonable efficiency while simultaneously providing enhanced bandwidth and miniaturization.

For the antenna with the $8-\mathrm{cm}$ square ground plane, the microstrip is $0.5-\mathrm{cm}$ wide, $L_{m}=0.58 \mathrm{~cm}$, and $d=1.2 \mathrm{~mm}$. The slot dimensions are 2.1 by $0.6 \mathrm{~cm}$. The simulated and measured return losses are shown in Fig. 8. The measured $10 \mathrm{~dB}$ bandwidth is $29 \%$; the simulated $10 \mathrm{~dB}$ bandwidth does not correspond to the merged resonance bandwidth. Disagreement between simulated and measured return loss is due to the reasons discussed in the $5.5 \mathrm{~cm}$ design. However, in this case, the problem of the air gap between the ground plane and the resonator is exacerbated due to the larger size of the flexible ground plane.

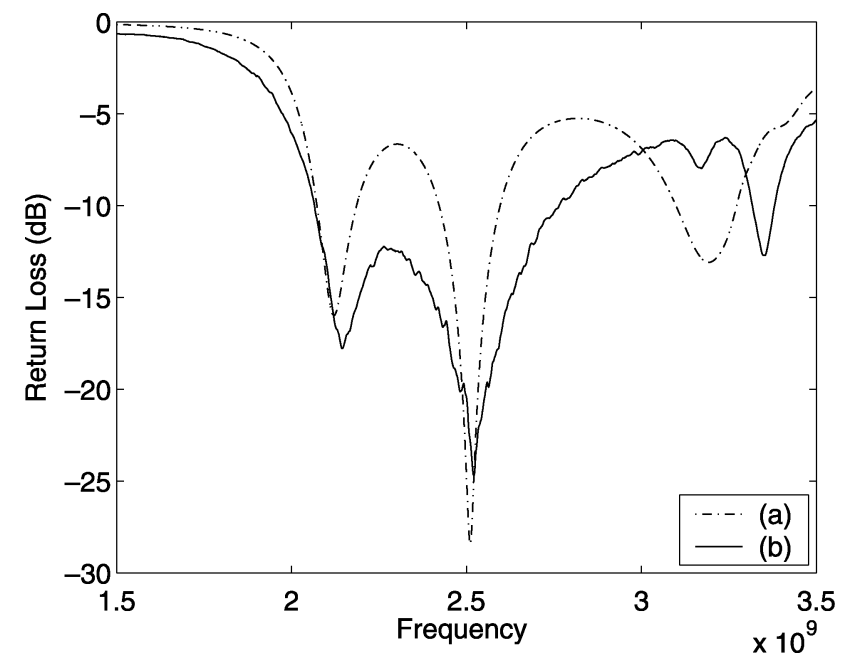

Fig. 8. Return loss for the 8-cm ground plane design (a) simulated and (b) measured.

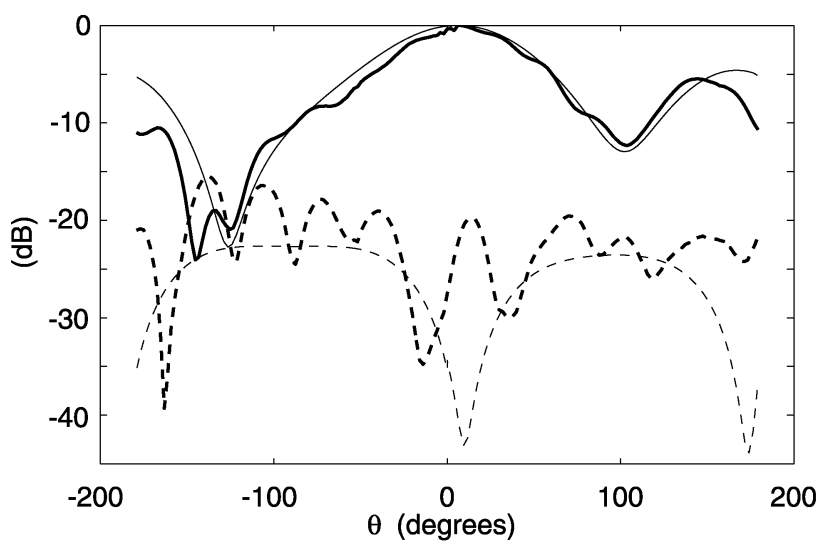

(a) Slot Resonance $f=2.17 \mathrm{GHz}$

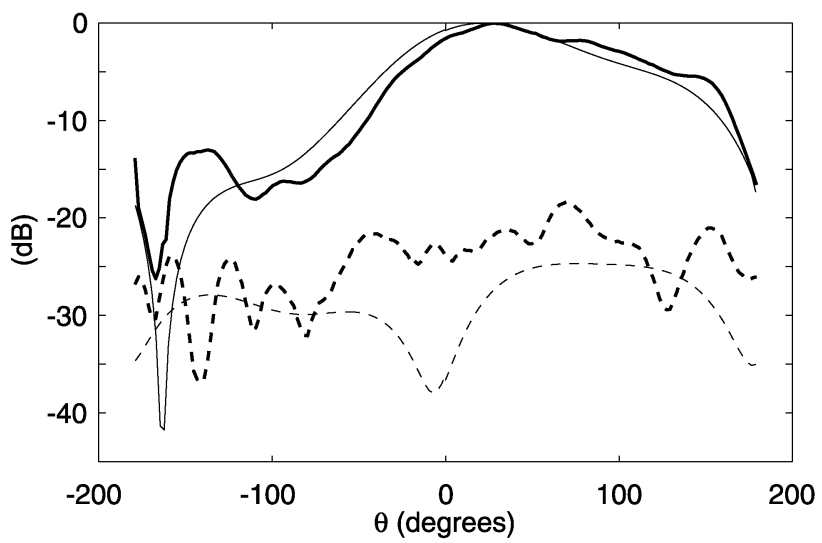

(b) DRA Resonance $f=2.57 \mathrm{GHz}$

Fig. 9. E-plane antenna patterns for the 8-cm ground plane design (a) $f=$ $2.17 \mathrm{GHz}$ and (b) $f=2.57 \mathrm{GHz}$. Measurements are shown in bold and simulations are shown in regular line weight.

Measured antenna patterns for the $8 \mathrm{~cm}$ design at both resonance frequencies in the E-plane are shown in Fig. 9; those of the H-plane are shown in Fig. 10. Again, the E-plane pattern asymmetry is, in part, due to the presence of the coaxial feed to the microstrip. Note the low cross-pol levels. As discussed in [6] and [20], the backlobe radiation usually present with a slot 


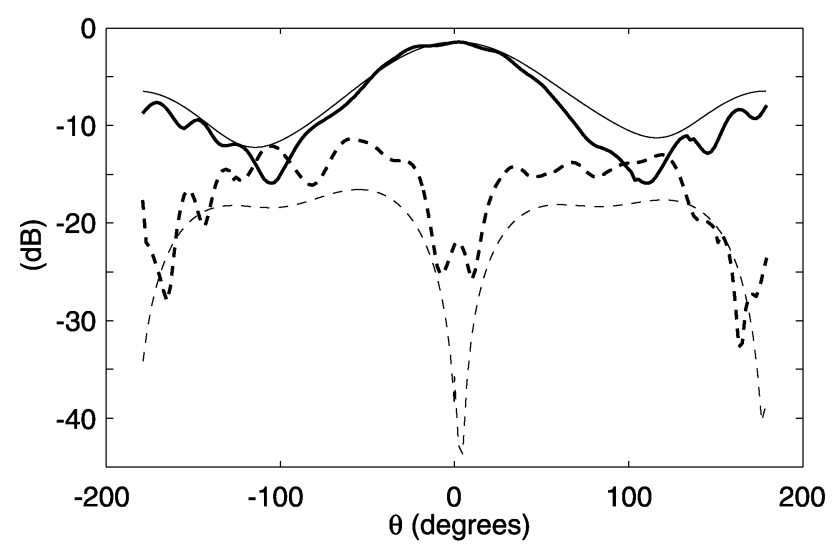

(a) Slot Resonance $f=2.17 \mathrm{GHz}$

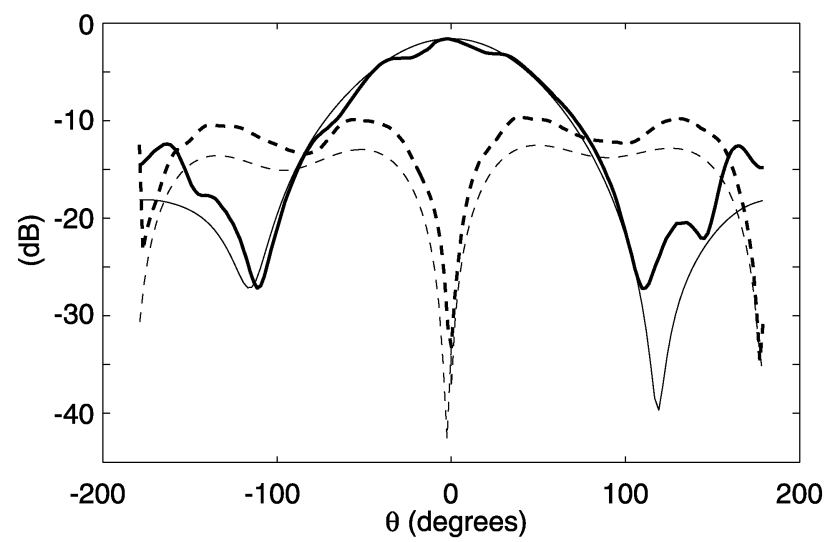

(b) DRA Resonance $f=2.57 \mathrm{GHz}$

Fig. 10. H-plane antenna patterns for the $8 \mathrm{~cm}$ ground plane design (a) $f=$ $2.17 \mathrm{GHz}$ and (b) $f=2.57 \mathrm{GHz}$. Measurements are shown in bold.

antenna is reduced by the DRA which radiates more effectively above the ground plane. This can be seen in the slot resonance frequency measurements which show less back radiation than would a regular slot antenna without the DRA.

The gain at the lower and upper resonant frequencies is approximately 4.6 and $4 \mathrm{dBi}$, respectively. Again using $D=2.7 \mathrm{~cm}$, the MAFM values are $0.41 \mathrm{~dB}$ and $-0.44 \mathrm{~dB}$ at the lower and upper resonance frequencies, respectively.

\section{CONCLUSION AND Future WORK}

It is shown that two resonances inherent to all DRA designs with resonant feed structures can be controlled for bandwidth optimization. A new, comprehensive figure of merit, or MAFM, is defined to allow comparison between various miniaturized antennas. Values obtained for the present antennas demonstrate that they provide enhanced bandwidth and miniaturization without degradation in gain. In addition, the similarity of the slot and DRA radiation characteristics allows preservation of the radiation patterns and polarization over the entire bandwidth. The design process is reviewed and the effects of varying design parameters are summarized. Two antennas exhibiting over $25 \%$ bandwidth and gain exceeding $4 \mathrm{dBi}$ are built and characterized.

Further increases in bandwidth may be obtained by incorporating the MSDRA technique referenced earlier and using a layer of higher permittivity dielectric on the bottom on the DRA, shifting the lower, slot resonance further down in frequency. Also, the bandwidth enhancing properties of magneto-dielectric metamaterials may be used with this design technique to yield extremely wide bandwidths [19], [24]. In simulation, 36\% bandwidths have been observed with a layered meta-material structure; work on the fabrication of DRAs using meta-materials is currently underway.

\section{REFERENCES}

[1] H. A. Wheeler, "Fundamental limits of small antennas," in Proc. IRE, vol. 35, Dec. 1947, pp. 1479-1484.

[2] M. W. McAllister, S. A. Long, and G. L. Conway, "The rectangular dielectric resonator," in Proc. Antennas and Propagation Soc. Int. Symp., vol. 21, May 1983, pp. 696-699.

[3] A. Kishk, A. Glisson, and G. Junker, "Bandwidth enhancement for split cylindrical dielectric resonator antennas," Progress in Electromagnetics Research, PIER 33, pp. 97-118, 2001.

[4] A. Kishk, Y. Yin, and A. Glisson, "Conical dielectric resonator antennas for wide-band applications," IEEE Trans. Antennas Propag., vol. 50, no. 4, pp. 469-474, Apr. 2002.

[5] K. W. Leung, K. Y. A. Lai, K. M. Luk, and D. Lin, "Input impedance of aperture coupled hemispherical dielectric resonator antenna," Electron. Lett., vol. 29, no. 13, pp. 1165-1167, 1993.

[6] R. K. Mongia and A. Ittipiboon, "Theoretical and experimental investigations on rectangular dielectric resonator antennas," IEEE Trans. Antennas Propag., vol. 45, no. 9, pp. 1348-1356, Sep. 1997.

[7] A. Ittipiboon, R. Mongia, Y. Antar, P. Bhartia, and M. Cuhaci, "Aperture fed rectangular and triangular dielectric resonators for use as magnetic dipole antennas," Electron. Lett., vol. 29, no. 23, pp. 2001-2002, 1993.

[8] J. Shin, A. Kishk, and A. Glisson, "Analysis of rectangular dielectric resonator antennas excited through a slot over a finite ground plane," in Proc. Antennas and Propagation Soc. Int. Symp., vol. 4, 2000, pp. 2076-2079.

[9] G. Junker, A. Kishk, and A. Glisson, "Input impedance of aperture-coupled dielectric resonator antennas," IEEE Trans. Antennas Propag., vol. 44, no. 5, pp. 600-607, May 1996.

[10] F. Croq and A. Papiernik, "Large bandwidth aperture coupled microstrip antenna," Electron. Lett., vol. 26, no. 16, pp. 1293-1294, 1990.

[11] A. Petosa, A. Ittipiboon, Y. M. M. Antar, D. Roscoe, and M. Cuhaci, "Recent advances in dielectric-resonator antenna technology," IEEE Antennas Propag. Mag., vol. 40, pp. 35-48, 1998.

[12] A. Kishk, "Wide-band truncated tetrahedron dielectric resonator antenna excited by a coaxial probe," IEEE Trans. Antennas Propag., vol. 51, no. 10, pp. 2913-2917, Oct. 2003.

[13] A. Ittipiboon, A. Petosa, D. Roscoe, and M. Cuhaci, "An investigation of a novel broadband dielectric resonator antenna," in Proc. Antennas and Propagation Society Int. Symp., vol. 3, 1996, pp. 2038-2040.

[14] A. Petosa, N. Simons, R. Siushansian, A. Ittipiboon, and M. Cuhaci, "Design and analysis of multisegment dielectric resonator antennas," IEEE Trans. Antennas Propag., vol. 48, no. 5, pp. 738-742, May 2000.

[15] M. H. Neshati and Z. Wu, "The determination of the resonance frequency of the $T E_{y}^{111}$ mode in a rectangular dielectric resonator for antenna applications,"' in Proc. 11th Int. Conf. Antennas and Propagation, 2001.

[16] R. K. Mongia, "Theoretical and experimental resonant frequencies of rectangular dielectric resonators," Proc. Inst. Elect. Eng., vol. 139, pp. 98-104, 1992

[17] P. L. Sullivan and D. H. Schaubert, "Analysis of an aperture coupled microstrip antenna," IEEE Trans. Antennas Propag., vol. 34, no. 8, pp. 977-984, Aug. 1986.

[18] C. Balanis, Advanced Engineering Electromagnetics. New York: Wiley, 1989.

[19] H. Mosallaei, "Complex layered materials and periodic electromagnetic band-gap structures: concepts, characterizations, and applications," Ph.D. dissertation, Univ. California, Los Angeles, 2001.

[20] Y. M. Antar and Z. Fan, "Theoretical investigation of aperture-coupled rectangular dielectric resonator antenna," Proc. Inst. Elect. Eng. on Microwaves, Antennas and Propagation, vol. 143, no. 2, pp. 113-118, Apr. 1996.

[21] G. Junker, A. Kishk, A. Glisson, and D. Kajfez, "Effect of fabrication imperfections for ground-plane-backed dielectric-resonator antennas," IEEE Antennas Propag. Mag., vol. 37, no. 1, pp. 40-47, 1995.

[22] _ _ "Effect of air gap on cylindrical dielectric resonator antenna operating in TM $_{01}$ mode," Electron. Lett., vol. 30, no. 2, pp. 97-98, 1994. 
[23] G. Drosses, Z. Wu, and L. Davis, "Aperture-coupled cylindrical dielectric resonator antenna," Microwave and Opt. Techno. Lett., vol. 20, no. 6, pp. 407-414, 1999.

[24] K. Sarabandi and H. Mosallaei, "Novel artificial embedded circuit meta-material for design of tunable electro-ferromagnetic permeability medium," in Proc. IEEE MTT-S Int. Microwave Symp. Dig. , vol. 3, 2003, pp. 2061-2064.

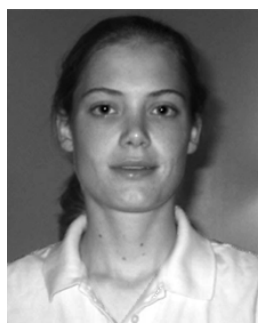

Amelia Buerkle (S'03) received the B.Sc. degree in electrical engineering from The Cooper Union, New York, in May 2002 and the M.S. degree in electrical engineering from the University of Michigan, Ann Arbor, in April 2004, where she is currently working towards the Ph.D. degree in the Radiation Laboratory.

Her current research interests include dielectric resonator antennas and metamaterials.

Ms. Buerkle is a recipient of a National Science Foundation (NSF) Graduate Research Fellowship.

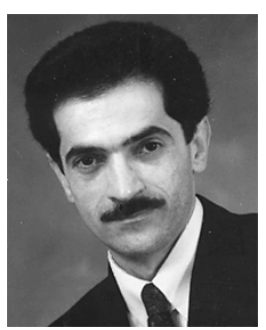

Kamal Sarabandi (S'87-M'90-SM'92-F'00) received the B.S. degree in electrical engineering from Sharif University of Technology, Tehran, Iran, in 1980, the M.S. degree in electrical engineering/mathematics, and the Ph.D. degree in electrical engineering from The University of Michigan-Ann Arbor, in 1986 and 1989, respectively.

$\mathrm{He}$ is Director of the Radiation Laboratory and a Professor in the Department of Electrical Engineering and Computer Science, The University of Michigan-Ann Arbor. He has 20 years of experience with wave propagation in random media, communication channel modeling, microwave sensors, and radar systems and is leading a large research group consisting of four research scientists, ten Ph.D. students, and two M.S. students. Over the past ten years he has graduated $20 \mathrm{Ph} . D$. students. He has served as the Principal Investigator on many projects sponsored by NASA, JPL, ARO, ONR, ARL, NSF, DARPA, and numerous industries. He has published many book chapters and more than 115 papers in refereed journals on electromagnetic scattering, random media modeling, wave propagation, antennas, microwave measurement techniques, radar calibration, inverse scattering problems, and microwave sensors. He has also had more than 230 papers and invited presentations in many national and international conferences and symposia on similar subjects. His research areas of interest include microwave and millimeter-wave radar remote sensing, electromagnetic wave propagation, and antenna miniaturization.

Dr. Sarabandi is a Member of the International Scientific Radio Union (URSI) Commission $\mathrm{F}$ and of The Electromagnetic Academy. He received the Henry Russel Award from the Regent of The University of Michigan-Ann Arbor (the highest honor the University of Michigan bestows on a faculty member at the assistant or associate level). He received a 1996 Teaching Excellence Award from the Department of Electrical Engineering and Computer Science and a 1999 GAAC Distinguished Lecturer Award from the German Federal Ministry for Education, Science, and Technology, given to about ten individuals worldwide in all areas of engineering, science, medicine, and law. He also received the 2003/2004 College of Engineering Research Excellence Award, The University of Michigan-Ann Arbor. In the past several years, joint papers presented by his students at a number of symposia (IEEE AP'95,'97,'00,'01,'03 IEEE IGARSS'99,'02, IEEE MTTS'01) have received student prize paper awards. He is a Vice President of the IEEE Geoscience and Remote Sensing Society (GRSS), a past Chairman of the Awards Committee of the IEEE GRSS from 1998 to 2002, and a Member of the IEEE Technical Activities Board Awards Committee from 2000 to 2002. He is an Associate Editor of the IEEE TRANSACTIONS ON ANTENNAS AND PROPAGATION and the IEEE SENSORS JOURNAL. He is listed in American Men \& Women of Science, Who's Who in America, and Who's Who in Electromagnetics.

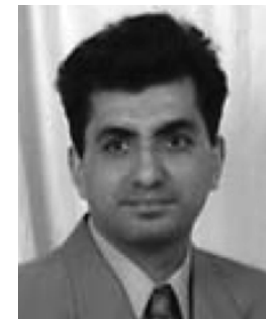

Hossein Mosallaei (SM'98) received the B.Sc. and M.Sc. degrees in electrical engineering from Shiraz University, Shiraz, Iran, and the Ph.D. degree in electrical engineering from the University of California, Los Angeles (UCLA), in 1991, 1994, and 2001, respectively.

From 1995 to 1997, he was with the Electrical Engineering Department at Shiraz University. From 1997 to 2001, he was a Research Assistant and Teaching Associate at UCLA. Currently, he is is a Research Scientist in the Department of Electrical Engineering and Computer Science, University of Michigan-Ann Arbor. His research interests include meta-materials, smart RF/wireless systems, novel communication antennas, micro-electromagnetics, periodic structures, EBG and photonic crystals, high frequency circuits, bioengineering, and computational EM.

Dr. Mosallaei is a Member of the International Scientific Radio Union (URSI) Commission B. He was the recipient of student prize paper awards in AP-S 2000, '01, '03, a URSI Young Scientist Award in 2001, and RMTG award in 2002. He was the organizer of Radiation Laboratory seminars at the University of Michigan-Ann Arbor in 2002 and 2003. He has also served as Chairman and Co-Chairman of several national and international symposia. He is listed in Who's Who in America. 